\title{
Spontaneous Spinal Subdural Hematoma Secondary to Hemophilia A and Zanubrutinib
}

\author{
John Lynes ${ }^{1}$ Sebastian Rubino ${ }^{1}$ Andrea Rogers ${ }^{1}$ Sameh Gaballa ${ }^{3}$ Hien D. Liu ${ }^{4}$ John A. Arrington ${ }^{2}$ \\ Edwin Peguero ${ }^{1}$ James K. C. Liu' ${ }^{1,50}$
}

${ }^{1}$ Department of Neuro-Oncology, H. Lee Moffitt Cancer Center and Research Institute, Tampa, Florida, United States

2 Department of Radiology, H. Lee Moffitt Cancer Center and Research Institute, Tampa, Florida, United States

${ }^{3}$ Department of Malignant Hematology, H. Lee Moffitt Cancer Center and Research Institute, Tampa, Florida, United States

${ }^{4}$ Department of Bone Marrow Transplant and Cellular

Immunotherapy, $\mathrm{H}$. Lee Moffitt Cancer Center and Research

Institute, Tampa, Florida, United States

${ }^{5}$ Department of Oncologic Sciences, University of South Florida

Morsani College of Medicine,. Tampa, Florida, United States
Address for correspondence James K. C. Liu, MD, Neurosurgical Oncology, Department of Neuro-Oncology, H. Lee Moffitt Cancer Center and Research Institute, Tampa, FL 33612, United States (e-mail: James.liu@moffitt.org).

J Neurol Surg Rep 2022;83:e19-e22.
Abstract
Keywords
- hemophilia
- zanubrutinib
- spinal subdural hematoma
- hemorrhage
- mantle cell lymphoma

Spontaneous spinal subdural hematomas (SSH) are rare occurrences that can occur most commonly secondary to vascular malformations or coagulopathies. Only a small fraction of spontaneous SSHs are caused by acquired coagulation disorders such as leukemia, hemophilia, and thrombocytopenia. This case report describes a patient with a history of Guillain-Barré syndrome (GBS), hemophilia A, and mantle cell lymphoma, on zanubrutinib therapy, a Bruton tyrosine kinase inhibitor associated with a risk of spontaneous hemorrhage. This patient developed a spontaneous spinal subdural hematoma, most likely due to the zanubrutinib therapy and exacerbated due to hemophilia. Treatment was delayed due to the patient's history of GBS that confounded the clinical diagnosis. This case is the first report of a spontaneous SSH in a patient on zanubrutinib, highlighting the need for a high index of suspicion for CNS hemorrhage in patients on Bruton's tyrosine kinase (BTK) inhibitor therapy.

\section{Introduction}

Spinal subdural hematomas (SSH) are a rare entity. Etiologies for SSH most often involve posttraumatic or iatrogenic causes although a subset of SSH are spontaneous secondary to vascular malformations or coagulopathies. ${ }^{1}$ Previous reviews have demonstrated that 10 to $35 \%$ of spontaneous SSH are secondary to anti-coagulation therapy, while 4 to $19 \%$ are secondary to acquired coagulation disorders such as leukemia, hemophilia, thrombocytopenia, polycythemia vera, and cryoglobulinemia. ${ }^{1-3}$

This case is the first reported spontaneous spinal subdural hematoma in a patient with a history of hemophilia A and mantle cell lymphoma on zanubrutinib treatment. The case was also unique in that in addition to the patient's acquired coagulation issues, the patient had a history of Guillain-Barré syndrome that resulted in an initial misdiagnosis before appropriate imaging was received

November 17, 2021

accepted

January 11, 2022
DOI https://doi.org/ 10.1055/a-1759-9841. ISSN 2193-6358. (c) 2022. The Author(s).

This is an open access article published by Thieme under the terms of the Creative Commons Attribution-NonDerivative-NonCommercial-License, permitting copying and reproduction so long as the original work is given appropriate credit. Contents may not be used for commercial purposes, or adapted, remixed, transformed or built upon. (https://creativecommons.org/ licenses/by-nc-nd/4.0/)

Georg Thieme Verlag KG, Rüdigerstraße 14, 70469 Stuttgart, Germany 
obtained and the patient was transferred for surgical intervention.

\section{Case Report}

A 76-year-old man with a history of hemophilia A and mantle cell lymphoma presented to an outside hospital for abdominal pain. He was initially diagnosed with mantle cell lymphoma (MCL) (TP53 mutated) 2 years ago by tissue biopsy, with CD5-positive monoclonal B cells identified by flow cytometry and t11;14 by fluorescence in situ hybridization (FISH). His hemophilia was mild with baseline factor VIII activity of $16 \%$ without inhibitor. His mantel cell lymphoma was diagnosed 2 years prior, with CD5-positive monoclonal B cells with 11:14 translocation identified by FISH. He underwent six cycles of rituximab-bendamustine, but was complicated by prolonged neutropenia requiring growth factor administration. Two months after completing bendamustine, he was admitted for neutropenic fever and diarrhea and diagnosed with Campylobacter colitis and bacteremia. Following this, he experienced ascending weakness involving all extremities, and was diagnosed with Guillain-Barré syndrome confirmed by EMG, requiring plasmapheresis and IVIG with one episode of relapse and ultimate complete neurological recovery. Twenty-two months following his initial diagnosis, he developed recurrence of his MCL and was started on zanubrutinib, at half dose due to his hemophilia A.

He had been on zanubrutinib for 4 months when he presented to the outside hospital, at which time he was diagnosed with gallstones with bile duct dilation and underwent endoscopic retrograde cholangiopancreatography. Forty-eight hours after presentation, the patient experienced ascending numbness and weakness involving the bilateral lower extremities and lower abdomen and midline back pain. Given his history of Guillain-Barré syndrome, he was treated with IVIG and steroids for presumed relapse. A spinal magnetic resonance imaging (MRI) was performed due to lack of improvement that demonstrated an intradural extramedullary lesion at the level of T10-11 with compression on the adjacent spinal cord ( - Fig. 1). This imaging also demonstrated subacute hemorrhagic fluid within the thecal sac extending caudally from T10 encasing the spinal cord and cauda equina. He was transferred to Moffitt Cancer Center for definitive neurosurgical evaluation.

At the time of transfer, the patent was an American Spinal Injury Association (ASIA) Grade B. He had no motor function in the lower extremities, a T10 sensory level, and upgoing Babinski reflexes. Laboratory studies on admission were significant for thrombocytopenia $(103 \mathrm{k} / \mu \mathrm{L})$ but normal factor VIII activity (74\%), PTT, and PT/INR. He was not hypertensive during his inpatient course.

The patient underwent T10-11 laminectomy and evacuation of subacute intradural hematoma causing the most significant compression of the spinal cord at T10 (-Fig. 2). No underlying vascular lesion or clear mass was identified. Postoperatively, the patient obtained relief of midline pain and improvement in sensation of the lower extremities, without significant changes in strength. In the perioperative period, the patient was administered von Willebrand factor every 12 hours to maintain normal Factor VIII activity and prevent hemorrhagic complications.

\section{Discussion}

Spontaneous spinal hematomas without underlying vascular or mass lesion are an uncommon pathologic entity. A systematic review of the literature reporting on 151 patients (ranging from 6 months to 87 years) with nontraumatic spontaneous acute spinal subdural hematomas found a small increased incidence between the first and second decades of life, with a major peak at the age of 60 years. ${ }^{3}$ In a different retrospective review of published reports of spontaneous SSH from 1948 to 2014, 122 cases were identified and $44 \%$ of cases was associated with the use of an anti-coagulant. ${ }^{2}$ Only $4 \%$ was associated with an acquired coagulation disorder, of which only one case was related to hemophilia. ${ }^{2}$ Separate reports of hemophilia A and $B$ has shown an association with spontaneous spinal hematomas, being reported in both pediatric and adult patients. $^{4-7}$ These have been reported in less than 0.2 to $0.8 \%$ of children with hemophilia also with abnormal coagulation cascade factor activity. ${ }^{8,9}$

The patient in this case had a complicated medical history that allowed several potential risk factors that could have contributed to his risk of spontaneous SSH. In addition, his history of Guillain-Barré syndrome, confounded the diagnosis that delayed definitive treatment. Although the patient's history of hemophilia puts him at a slight risk for spontaneous SSH, as demonstrated in previous reviews, it is likely that zanubrutinib played a strong role in his risk of spontaneous hemorrhage.

Zanubrutinib, a second-generation Bruton's tyrosine kinase (BTK) inhibitor, is used as a treatment of relapsing mantle cell lymphoma (MCL). BTK is expressed on B lymphocytes, myeloid cells, and platelets. However, BTK inhibitors can have "off-target" kinase inhibitions (e.g., EGFR, TEC, and Src family kinases) that can lead to certain toxicities (e.g., atrial fibrillation, skin rash, and diarrhea). The pathophysiology of increased bleeding risk associated with BTK inhibitors is currently not well understood and is thought to be mediated by inhibiting platelet activation, aggregation, and thrombus formation possibly through the inhibition of Src family kinases. ${ }^{10,11}$ Second-generation BTK inhibitors, including zanubrutinib, were developed to more selectively target BTK while exhibiting less off-target activity, decreasing the adverse side-effect profile. ${ }^{12} \mathrm{~A}$ study of zanubrutinib for MCL reported $9.4 \%$ bleeding adverse events though none within the central nervous system, while a recent review found a range of reported bleeding events in 3 to $36 \%$ of patients on BTK inhibitor therapy for B cell malignancies. ${ }^{13,14}$ Though hemorrhage is a consistently reported adverse reaction of zanubrutinib, CNS involvement appears extraordinarily rare without any reported cases of spontaneous spinal hematomas. $^{12}$ 

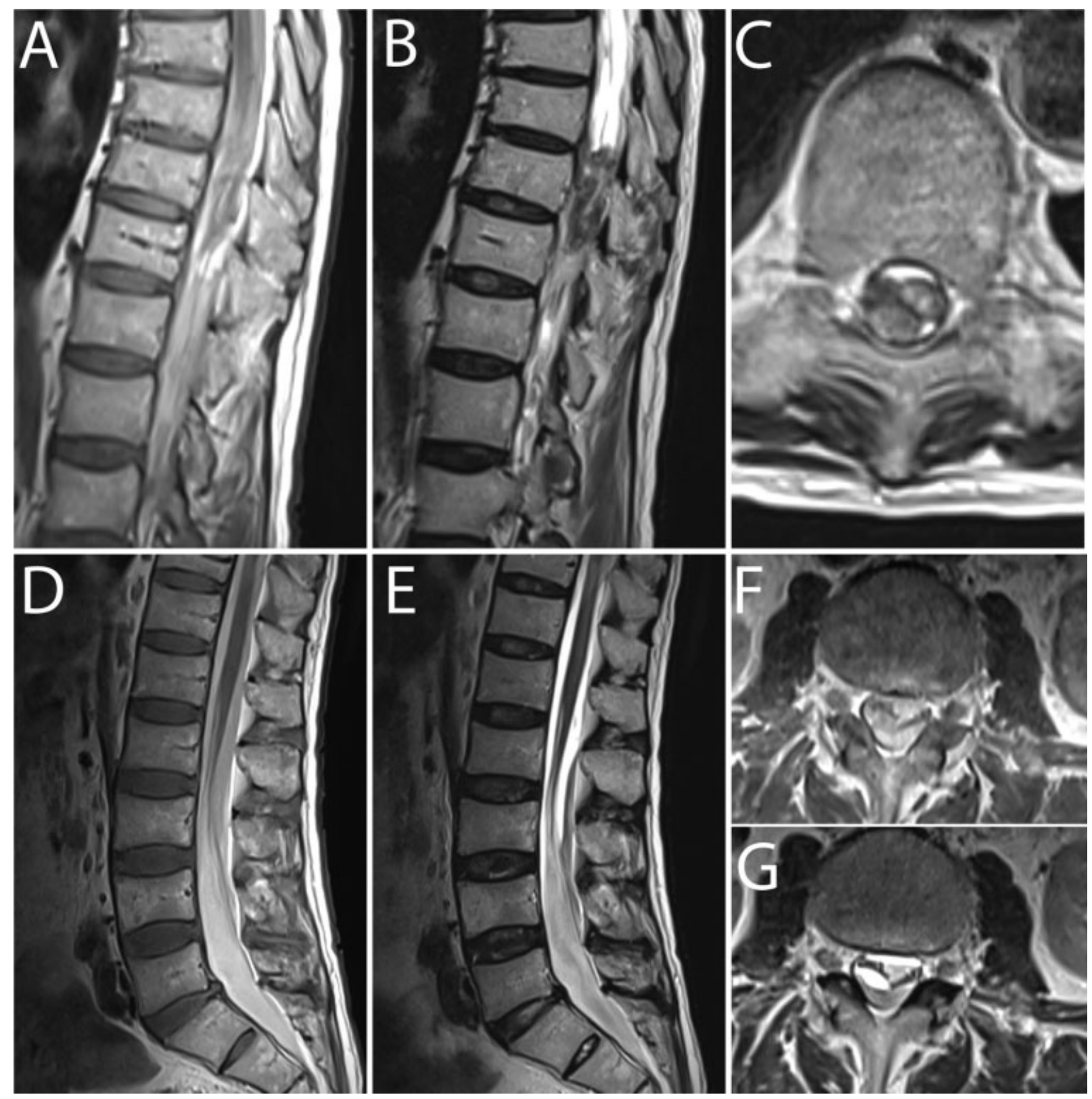

Fig. 1 (A) Preoperative sagittal T1-weighted image of the thoracic spine, demonstrating extensive isointense and hyperintense signal reflecting intrathecal blood products. (B) Preoperative sagittal and (C) axial T2-weighted image of the thorax, demonstrating dorsolateral acute subdural hematoma with greatest degree of spinal cord compression at the level of T10. (D, E) Preoperative sagittal T1-weighted and T2-weighted images showing extensive, relatively homogenously hyperintense acute blood through the distal lumbosacral intradural space. (F, G) Preoperative axial T1-weighted and $\mathrm{T} 2$-weighted sequences of the level of $\mathrm{L} 5$, demonstrating casting of the thecal sac by blood products with clumping of spinal nerve roots encased in hematoma.

Although the etiology for the spontaneous SSH in this case is likely a combination of hemophilia and zanubrutinib, it is unclear as to whether there may have been an inciting event that led to the hemorrhage 48 hours following admission to the outside hospital and an ERCP procedure. Possibilities may include an occult trauma to the spine or a hypertensive episode secondary to pain following the procedure. In this case, diagnosis was delayed due to the confounding history of Guillain-Barré syndrome although the presentation was not consistent in that both motor and sensory functions were impaired. This scenario highlights the need for awareness of acute central nervous systemrelated hemorrhages in a patient with an underlying acquired coagulation disorder while on a medication with a risk for hemorrhage. Acute onset of these symptoms should raise suspicion for an acute neurological event that warrants immediate work-up that should include MRI of the spine.

In addition to spontaneous $\mathrm{SSH}$, the risk of iatrogenic $\mathrm{SSH}$ is elevated as a nearly half of previously spontaneous SSH are secondary to iatrogenic causes, such as lumbar puncture, with an underlying coagulopathy. ${ }^{3}$ Therefore, the risk of a 

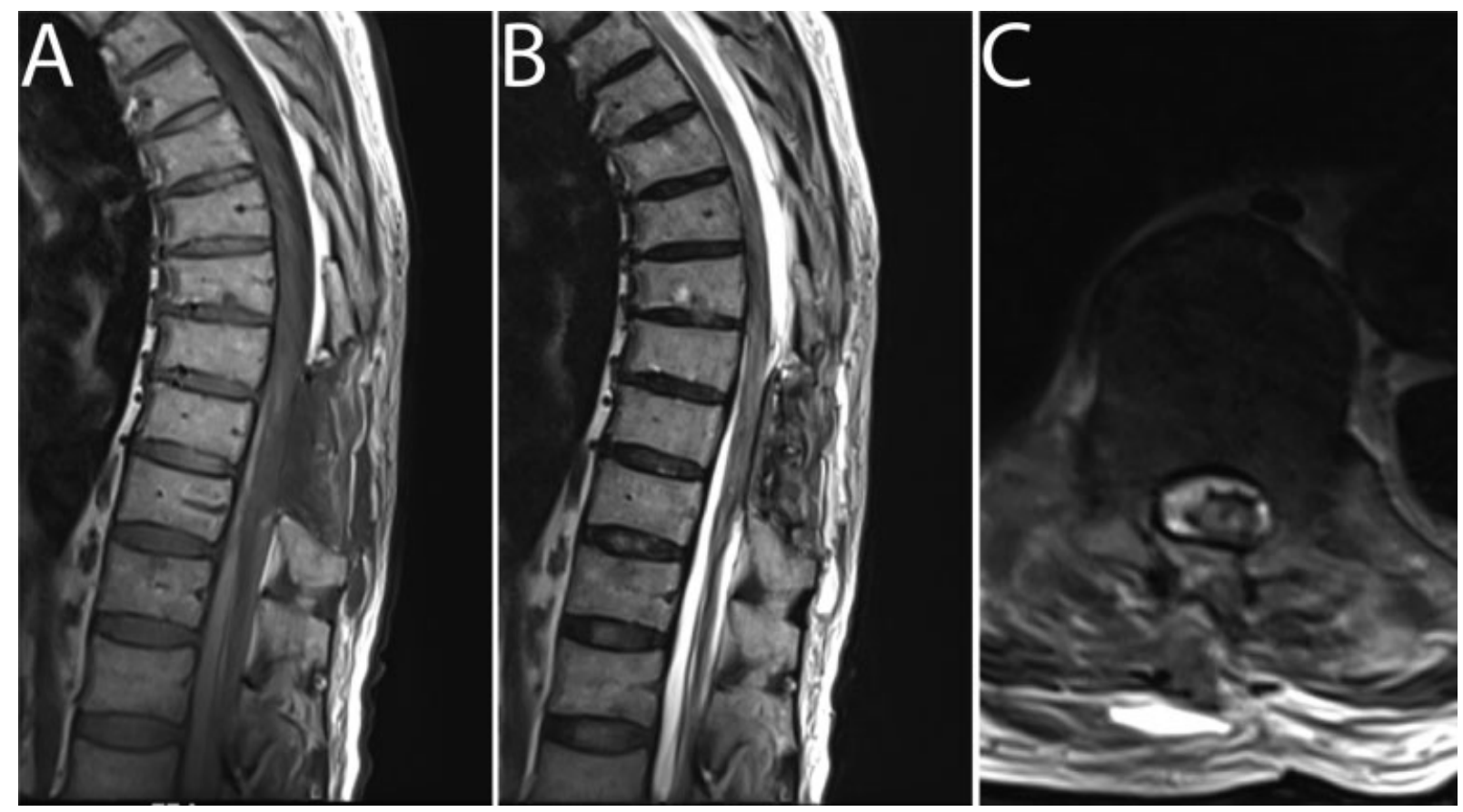

Fig. 2 (A) Postoperative sagittal T1-weighted MRI sequence image of the thorax, demonstrating evacuation of focal hematoma. (B, C) Postoperative sagittal and axial T2-weighted MRI sequence image of the thorax, demonstrating circumferential hyperintense signal of CSF surrounding the spinal cord, reflecting effective decompression.

SSH needs to be accounted for when considering invasive procedures on these patients.

\section{Conclusion}

Spontaneous spinal subdural hematomas can occur in the presence of acquired coagulation disorders, such as hemophilia and BTK inhibitor therapy. This case highlights the need for maintaining a high index of suspicion of spontaneous hemorrhage in the central nervous system while on this type of therapy.

\section{Conflict of Interest \\ None declared.}

\section{References}

1 Domenicucci M, Ramieri A, Ciappetta P, Delfini R. Nontraumatic acute spinal subdural hematoma: report of five cases and review of the literature. J Neurosurg 1999;91(1, Suppl)65-73

2 de Beer MH, Eysink Smeets MM, Koppen H. Spontaneous spinal subdural hematoma. Neurologist 2017;22(01):34-39

3 Pereira BJ, de Almeida AN, Muio VM, de Oliveira JG, de Holanda $\mathrm{CV}$, Fonseca NC. Predictors of outcome in nontraumatic spontaneous acute spinal subdural hematoma: case report and literature review. World Neurosurg 2016;89:574-577.e7

4 Rathi N, Rathi A. Haemophilia A presenting as spontaneous spinal epidural haematoma in a 3-month-old infant. BMJ Case Rep 2010; 2010:bcr0520102997
5 Nirupam N, Pemde H, Chandra J. Spinal epidural hematoma in a patient with hemophilia B presenting as acute abdomen. Indian J Hematol Blood Transfus 2014;30(Suppl 1):54-56

6 Ahn DK, Jung WS, Lee JI. Hemophilia A in a senior patient: a case report of spinal epidural hematoma as first presentation. Asian Spine J 2015;9(03):452-455

7 Meena AK, Jayalakshmi S, Prasad VS, Murthy JM. Spinal epidural haematoma in a patient with haemophilia-B. Spinal Cord 1998;36 (09):658-660

8 Eyster ME, Gill FM, Blatt PM, Hilgartner MW, Ballard JO, Kinney TR. Central nervous system bleeding in hemophiliacs. Blood 1978;51(06):1179-1188

9 Kiehna EN, Waldron PE, Jane JA. Conservative management of an acute spontaneous holocord epidural hemorrhage in a hemophiliac infant. J Neurosurg Pediatr 2010;6(01):43-48

10 von Hundelshausen P, Siess W. Bleeding by Bruton tyrosine kinase-inhibitors: dependency on drug type and disease. Cancers (Basel) 2021;13(05):1103

11 Senis YA, Mazharian A, Mori J. Src family kinases: at the forefront of platelet activation. Blood 2014;124(13):2013-2024

12 Song Y, Zhou K, Zou D, et al. Treatment of patients with relapsed or refractory mantle-cell lymphoma with zanubrutinib, a selective inhibitor of Bruton's tyrosine kinase. Clin Cancer Res 2020;26 (16):4216-4224

13 Tam CS, Opat S, Simpson D, et al. Zanubrutinib for the treatment of relapsed or refractory mantle cell lymphoma. Blood Adv 2021;5 (12):2577-2585

14 Wang J, Zhao A, Zhou H, Zhu J, Niu T. Risk of bleeding associated with ibrutinib in patients with B-cell malignancies: a systematic review and meta-analysis of randomized controlled trials. Front Pharmacol 2020;11:580622 